\title{
Phase stability and the arsenic vacancy defect in $\operatorname{In}_{x} \mathrm{Ga}_{1-x} \mathrm{As}$
}

\author{
S. T. Murphy, A. Chroneos, and R. W. Grimes \\ Department of Materials, Imperial College London, London SW7 2AZ, United Kingdom
}

C. Jiang

Materials Science and Technology Division, Los Alamos National Laboratory, Los Alamos, New Mexico 87545, USA

U. Schwingenschlögl

PSE Division, KAUST, Thuwal 23955-6900, Kingdom of Saudi Arabia

(Received 13 September 2011; revised manuscript received 19 October 2011; published 17 November 2011; corrected 18 November 2011)

\begin{abstract}
The introduction of defects, such as vacancies, into $\operatorname{In}_{x} \mathrm{Ga}_{1-x}$ As can have a dramatic impact on the physical and electronic properties of the material. Here we employ ab initio simulations of quasirandom supercells to investigate the structure of $\operatorname{In}_{x} \mathrm{Ga}_{1-x}$ As and then examine the energy and volume changes associated with the introduction of an arsenic vacancy defect. We predict that both defect energies and volumes for intermediate compositions of $\operatorname{In}_{x} \mathrm{Ga}_{1-x}$ As differ significantly from what would be expected by assuming a simple linear interpolation of the end member defect energies/volumes.
\end{abstract}

DOI: $10.1103 /$ PhysRevB.84.184108

PACS number(s): $71.55 . \mathrm{Eq}$

\section{INTRODUCTION}

In recent years the $\operatorname{In}_{x} \mathrm{Ga}_{1-x}$ As system has drawn significant interest due to a myriad of potentially useful applications. These include use as a material for $n$-channel metal-oxide field-effect transistors ( $n$-MOSFETS $)^{1,2}$ and as a bottom cell material in multijunction solar cells. ${ }^{3}$ Additionally, the efficient manipulation of quantum dots (QD) using straindriven epitaxy has opened up the possibility of applications in optoelectronic devices such as high-efficiency QD lasers and single or multicolored QD photodetectors. ${ }^{4}$

$\mathrm{In}_{x} \mathrm{Ga}_{1-x}$ As can be formed from the reaction of GaAs and InAs as shown below:

$$
x \mathrm{InAs}+(1-x) \mathrm{GaAs} \rightarrow \operatorname{In}_{x} \mathrm{Ga}_{1-x} \mathrm{As},
$$

where GaAs, InAs, and $\operatorname{In}_{x} \mathrm{Ga}_{1-x}$ As exhibit the zincblende structure with the $F \overline{4} 3 m$ (216) space group. In the binary alloys As atoms are assigned to the $4 c$ Wyckoff sites with the $\mathrm{Ga}$ or In atoms residing on the $4 a$ Wyckoff sites, thus forming two interpenetrating face-centered cubic (FCC) lattices. On formation of the ternary alloy, the As atoms remain restricted to the $4 c$ sublattice while the $\mathrm{Ga}$ and In atoms are randomly distributed on the $4 a$ sites. In the binary alloys each atom is tetrahedrally coordinated with point group symmetry $T_{d}$; however, the random distribution of atoms on the $4 a$ sublattice in the ternary alloy leads to local distortions that break the perfect tetrahedral site symmetry.

Point defects, such as vacancies and interstitials, are known to play a pivotal role in the accommodation of dopants and diffusion of both intrinsic and extrinsic species through a crystal matrix. Consequently, defects in semiconductors have been studied intensively, with particular attention focused on $\mathrm{Si}$ and GaAs. While point defects in GaAs have been explored extensively using $a b$ initio simulation techniques, the remainder of the $\operatorname{In}_{x} \mathrm{Ga}_{1-x}$ As system appears to have been largely neglected. In part, this is due to the difficulty in modeling a random alloy.

One of the earliest attempts to quantify the defect formation energy of an As vacancy in the $\operatorname{In}_{x} \mathrm{Ga}_{1-x}$ As system was by
Van Vechten, ${ }^{5}$ who approximated the formation energy of the charge-neutral defects in the end member binary alloys to the surface energy of the cavity. Values of 2.59 and $2.07 \mathrm{eV}$ were obtained for GaAs and InAs, respectively. These initial calculations made a number of assumptions regarding the shape and, therefore, the type of surface of the cavity. Density-functional theory (DFT) simulations employing energy minimization do not require such approximations to be made as the atomic relaxation is determined explicitly.

A number of groups have observed a Jahn-Teller distortion of the gallium atoms surrounding the charge-neutral arsenic vacancy defect in $\mathrm{GaAs}^{6-8}$ in DFT simulations. The $T_{d}$ symmetry is lost due to the formation of two bonding orbitals between the pairs of nearest-neighbor Ga atoms resulting in a $D_{2 d}$ symmetry. This is similar to the distortion surrounding the $\mathrm{V}_{\mathrm{Si}}^{0}$ and $\mathrm{V}_{\mathrm{Si}}^{+1}$ defects in silicon predicted using DFT. ${ }^{9,10}$ El-Mellouhi and Mousseau ${ }^{6}$ report a formation energy of $3.25 \mathrm{eV}$ for the Jahn-Teller distorted $\mathrm{V}_{\mathrm{As}}^{0}$ defect (under As-rich conditions).

There is some discrepancy in the literature concerning the stability of the $\mathrm{V}_{\mathrm{As}}^{0}$ defect. Several studies predict that $\mathrm{V}_{\mathrm{As}}^{0}$ is a negative- $U$ state (i.e., it is more energetically favorable to transfer two electrons at the same time to the defect level from the Fermi level via $\mathrm{V}_{\mathrm{As}}^{+}+2 e^{-} \rightarrow \mathrm{V}_{\mathrm{As}}^{-}$rather than $\mathrm{V}_{\mathrm{As}}^{+}+$ $\left.e^{-} \rightarrow \mathrm{V}_{\mathrm{As}}^{0}\right), 6,7,11,12$ while others, including the most recent results calculated using hybrid functionals, claim that $\mathrm{V}_{\mathrm{As}}^{0}$ is stable. ${ }^{13-15}$ There is less controversy surrounding the stability of the $\mathrm{V}_{\mathrm{As}}^{0}$ defect in InAs which is also predicted to be a metastable negative- $U$ state, although it is predicted to be stable at a (100) surface. ${ }^{16}$ The formation energy of the vacancy in the bulk is not given in this work; however, the value must be $>2.6 \mathrm{eV}$ to allow $\mathrm{V}_{\mathrm{As}}^{0}$ to be a negative- $U$ state. $^{16}$

In the intermediate regime, the photoluminescence study of Gillin et al. ${ }^{17}$ suggests that the formation energy for an As vacancy defect in $\mathrm{In}_{0.66} \mathrm{Ga}_{0.33} \mathrm{As}$ is approximately $2.0 \mathrm{eV}$. To the best of our knowledge, there are no formation energies for the arsenic vacancy in any other composition of $\operatorname{In}_{x} \mathrm{Ga}_{1-x} \mathrm{As}$ and certainly no relationship between the defect formation energy and $x$ has been established. Therefore, in this article we 
employ DFT simulations to investigate the thermodynamics of the $\operatorname{In}_{x} \mathrm{Ga}_{1-x}$ As system and compare our results with the thermodynamic data from the literature before moving on to examine the formation energy of the arsenic vacancy defect as a function of InAs concentration (i.e., of $x$ ).

\section{METHODOLOGY}

\section{A. Density-functional theory simulations}

All DFT simulations presented here were conducted using the CASTEP 5.5 simulation package. ${ }^{18,19}$ CASTEP is a planewave pseudopotential code that describes a crystal using supercells and periodic boundary conditions with special point integration over the Brillouin zone. Exchange correlation was described using the generalized gradient approximation of Perdew, Burke, and Ernzerhof (GGA-PBE). ${ }^{20}$ A Monkhorst$\mathrm{Pack}^{21}$ scheme is used to sample the Brillouin zone with a density of $\approx 0.03 \AA^{-1}$ on each axis, corresponding to $3 \times 3 \times 3$ and $2 \times 2 \times 2 k$-point grids for the 64 and 216 atom supercells, respectively. Pseudopotentials were developed using the "onthe-fly" pseudopotential generating feature in CASTEP and the smoothing parameters were optimized following Murphy et al. ${ }^{22}$ Semicore $3 d$ and $4 d$ electrons are treated as valence for $\mathrm{Ga}$ and $\mathrm{In}$, respectively, and the plane-wave cutoff energy was set to $380 \mathrm{eV}$, offering a precision of $10^{-2} \mathrm{eV}$ per formula unit. Finally, the Fourier transform grid for the electron density is larger than that of the wave functions by a scaling factor of 2.0 (represented in CASTEP using the grid_scale parameter).

To ensure that the optimum precision was obtained, we used a convergence criterion for the self-consistent simulations of $5 \times 10^{-6} \mathrm{eV}$. Similarly, robust criteria for the geometry optimization were employed as follows: energy $=2 \times$ $10^{-5} \mathrm{eV}$ atom $^{-1}$, forces $=0.05 \mathrm{eV} / \AA$, stress $=0.1 \mathrm{GPa}$, and displacement $=2 \times 10^{-3} \AA$.

Five compositions of $\operatorname{In}_{x} \mathrm{Ga}_{1-x}$ As have been studied corresponding to $x=0.0,0.25,0.5,0.75$, and 1.0 , where $x=$ 0.0 and 1.0 correspond to the end members GaAs and InAs, respectively. The arsenic vacancy defect was selected for investigation here due to it being the only vacancy defect that can exist across the entire compositional range.

\section{B. Special quasirandom structures}

In order to model a disordered system, such as the $4 a$ sublattice in $\operatorname{In}_{x} \mathrm{Ga}_{1-x} \mathrm{As}$, while still retaining full atomistic detail and, thus, including all local relaxation effects, Zunger et $a l .{ }^{23}$ have developed a series of special quasirandom supercells (SQS). These small unit cell structures closely mimic the most relevant near-neighbor pair and multisite correlation functions of random alloys. The efficacy of DFT simulations employing SQS cells has been established by Haas et $a l .{ }^{24}$ The simulations presented here employed 64 atom supercells based on the SQS methodology and are the same as those used in our previous work ${ }^{25}$ (more details, including pictorial representations of the supercells, can be found in this previous work). This combination of DFT simulations and SQS supercells has been used previously to examine the stability of $E$ centers in $\mathrm{Si}_{1-x} \mathrm{Ge}_{x} \cdot{ }^{26}$

\section{Defect formation formalism}

Within the supercell methodology, the formation of a defect can be considered as exchange between the host material and some atomic and/or electronic reservoir. Following the formalism of Zhang and Northup ${ }^{27}$ the formation energy of a defect is given by

$$
E_{F}=E_{\mathrm{DFT}}(D)-E_{\mathrm{DFT}}\left(\operatorname{In}_{x} \mathrm{Ga}_{1-x} \mathrm{As}\right)+\sum_{i} n_{i} \mu_{i}+q_{D} E_{f},
$$

where $E_{\mathrm{DFT}}(D)$ and $E_{\mathrm{DFT}}\left(\operatorname{In}_{x} \mathrm{Ga}_{1-x} \mathrm{As}\right)$ are the DFT total energies of $\operatorname{In}_{x} \mathrm{Ga}_{1-x}$ As with and without the defect, $D ; n_{i}$ is the number of atoms removed/added (in this convention addition of an atom would result in a negative value for $n_{i}$ ); $\mu_{i}$ is the chemical potential of the atoms removed; $q$ is the charge on the defect; and $E_{f}$ is the Fermi energy. As this study is limited to the investigation of charge-neutral defects, the final term in Eq. (2) can be neglected. Furthermore, it is not necessary to employ any post-simulation corrections normally associated with the calculation of defect formation energies of charged defects in semiconductors. ${ }^{28}$

\section{RESULTS AND DISCUSSION}

\section{A. Elemental solids}

Single unitcells of the elemental In, Ga, and As were relaxed to the criteria described in Sec. II A. Table I shows that there is excellent agreement between the lattice parameters predicted by our simulations and the experimental values. The DFT values are slightly larger than the experimental values, which is common for simulations employing the GGA exchange correlation functional.

Atomization energies were determined using Eq. (3) as follows:

$$
E_{a}=E\left(\operatorname{In}_{i} \mathrm{Ga}_{j} \mathrm{As}_{k}\right)-[i E(\mathrm{In})+j E(\mathrm{Ga})+k E(\mathrm{As})],
$$

where $E\left(\operatorname{In}_{i} \mathrm{Ga}_{j} \mathrm{As}_{k}\right)$ is the energy per formula unit of $\mathrm{In}_{i} \mathrm{Ga}_{j} \mathrm{As}_{k}$ (for example, if $j=k=0$, this corresponds to elemental $\mathrm{In}) . E(\mathrm{In}), E(\mathrm{Ga})$, and $E(\mathrm{As})$ are the reference

TABLE I. Relaxed lattice parameters and atomization energies for elemental In, $\mathrm{Ga}$, and As compared to experimental data from the literature (no correction for zero temperature vibrational energy has been included in the DFT atomization energies).

\begin{tabular}{lcrr}
\hline \hline Element & Property & DFT & Experiment \\
\hline In & $\mathrm{a} / \AA$ & 3.23 & $3.25^{29}$ \\
I4/MMM & $\mathrm{c} / \AA$ & 5.28 & $4.95^{29}$ \\
& Volume $/ \AA^{3}$ & 54.91 & $52.26^{29}$ \\
& $E_{a} / \mathrm{eV}$ & 2.32 & $2.26^{30}$ \\
Ga & $\mathrm{a} / \AA$ & 2.90 & $2.81^{31}$ \\
I4/MMM & $\mathrm{c} / \AA$ & 4.57 & $4.46^{31}$ \\
& Volume $/ \AA^{3}$ & 38.34 & $35.15^{31}$ \\
& $E_{a} / \mathrm{eV}$ & 2.61 & $2.81^{32}$ \\
As & $\mathrm{a} / \AA$ & 3.81 & $3.76^{33}$ \\
R3-MH & $\mathrm{c} / \AA$ & 10.62 & $10.55^{33}$ \\
& Volume $/ \AA^{3}$ & 133.18 & $129.12^{33}$ \\
& $E_{a} / \mathrm{eV}$ & 3.01 & $3.12^{30}$ \\
\hline \hline
\end{tabular}


states of the constituent atoms modeled as single atoms in a monoclinic cell with $a=15.79 \AA, b=15.50 \AA, c=15.00 \AA$, and $\beta=98.75^{\circ}$ with periodic boundary conditions. Spinpolarized calculations used 1, 1, and 3 unpaired electrons for In, $\mathrm{Ga}$, and As atoms, respectively. The atomization energies for the elemental solids are in good agreement with the experimental data; consequently, the values for the chemical potentials of the constituent elements of $\operatorname{In}_{x} \mathrm{Ga}_{1-x}$ As in their elemental state are expected to be accurate.

\section{B. Structure and thermodynamics of $\operatorname{In}_{x} G a_{1-x} A s$}

Figure 1 shows a plot of the relaxed lattice parameter as a function of the mole fraction of InAs in $\operatorname{In}_{x} \mathrm{Ga}_{1-x}$ As (i.e., $x$ ). The dashed line in Fig. 1 represents a linear interpolation of the lattice parameters of the end-member binary alloys corresponding to ideal mixing (or Vegard's law). ${ }^{34}$ Lattice parameters for intermediate compositions of $\operatorname{In}_{x} \mathrm{Ga}_{1-x}$ As are predicted to be very slightly lower than would be expected from a linear interpolation of the end-member binary alloys as suggested for this and other ternary III-V alloys in our previous work. ${ }^{25}$ As was the case for the elemental solids, the DFT lattice parameters of $\operatorname{In}_{x} \mathrm{Ga}_{1-x}$ As are slightly larger than the experimental values (see Fig. 1); however, in general, the agreement is good.

The atomization energy of $\operatorname{In}_{x} \mathrm{Ga}_{1-x}$ As as a function of $x$ is given in Fig. 2. To the best of our knowledge, there are no experimental data available for the atomization energies of $\mathrm{In}_{x} \mathrm{Ga}_{1-x}$ As at $0 \mathrm{~K}$; however, Paier et al. ${ }^{39}$ use experimental data measured at $298.15 \mathrm{~K}$ to derive a value of $6.62 \mathrm{eV}$ for the $0 \mathrm{~K}$ atomization of a formula unit of GaAs. This value is in good agreement with the $6.34 \mathrm{eV}$ determined here (Paier et al. also calculated an atomization energy for GaAs of 6.20 eV using the B3LYP). The dashed line in Fig. 2 represents ideal mixing and it is evident that the atomization energies for intermediate values of $x$ deviate from ideal solution.

Assuming that $\operatorname{In}_{x} \mathrm{Ga}_{1-x}$ As can be formed from the reaction of InAs and GaAs via reaction (1), the enthalpy of mixing,

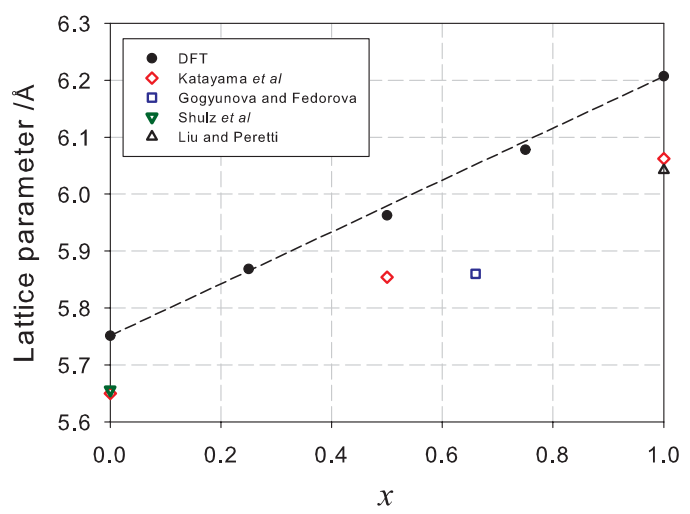

FIG. 1. (Color online) Plot showing the change in the lattice parameter of $\operatorname{In}_{x} \mathrm{Ga}_{1-x}$ As as a function of the $x$ calculated using DFT and the SQS supercells. Included for comparison is the experimental data of Katayama et al., ${ }^{35}$ Gogyunova and Fedorova, ${ }^{36}$ Liu and Peretti, ${ }^{37}$ and Schulz et al. ${ }^{38}$

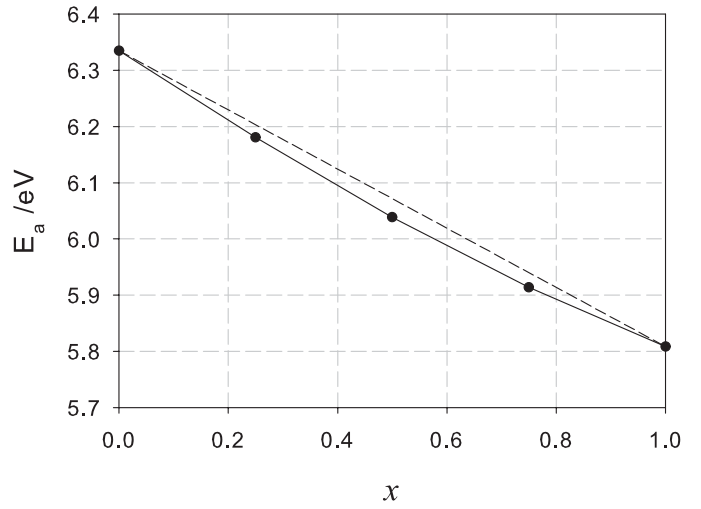

FIG. 2. Plot showing the atomization energy of $\operatorname{In}_{x} \mathrm{Ga}_{1-x}$ As as a function of $x$.

$\Delta H_{m}$, can be determined using Eq. (4),

$$
\Delta H_{m}=E\left(\operatorname{In}_{x} \mathrm{Ga}_{1-x} \mathrm{As}\right)-[x E(\mathrm{InAs})+(1-x) E(\mathrm{GaAs}],
$$

where $E\left(\operatorname{In}_{x} \mathrm{Ga}_{1-x} \mathrm{As}\right), E(\mathrm{InAs})$, and $E(\mathrm{GaAs})$ are the lattice energies of $\operatorname{In}_{x} \mathrm{Ga}_{1-x}$ As, InAs, and GaAs, respectively. The enthalpy of mixing is plotted as a function of $x$ in Fig. 3 and shows a small positive enthalpy of mixing across the entire composition range, in agreement with the experimental data of Rugg et al., ${ }^{40}$ hinting at the presence of a miscibility gap in the GaAs-InAs phase diagram.

If the free energy, $\Delta G$, of reaction (1) is positive, then the $\mathrm{In}_{x} \mathrm{Ga}_{1-x}$ As will undergo phase separation into distinct regions of InAs and $\mathrm{GaAs}^{41}{ }^{4}$ a process that is limited by the diffusion rate of the atoms. The Gibb's free energy can be calculated using

$$
\Delta G=\Delta H_{m}-T \Delta S,
$$

where $T$ is the temperature and $\Delta S$ is the entropy associated with reaction (1). For an alloy, such as $\operatorname{In}_{x} \mathrm{Ga}_{1-x} \mathrm{As}$, where the In and As atoms are randomly distributed on the $4 a$ Wyckoff sites, the configurational entropy can be determined by

$$
\Delta S=k_{B} \ln \Omega,
$$

where $k_{B}$ is the Boltzmann constant and $\Omega$ is the number of ways of arranging $x$ moles of In and $1-x$ moles (where

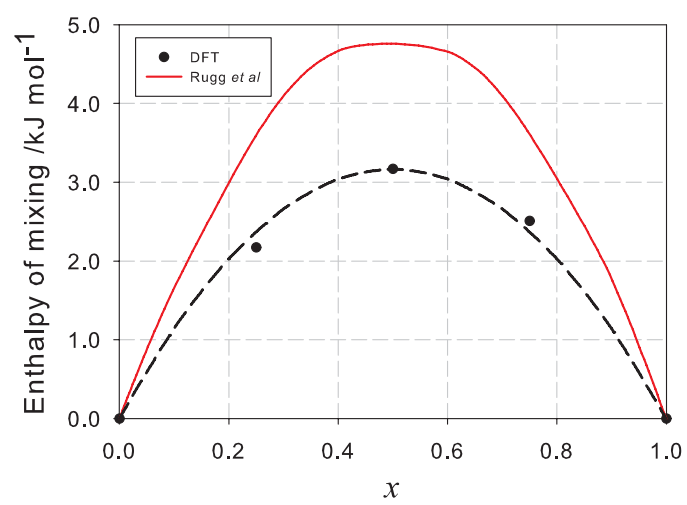

FIG. 3. (Color online) Plot showing the enthalpy of mixing of $\mathrm{In}_{x} \mathrm{Ga}_{1-x}$ As as a function of $x$ compared to the experimental data of Rugg et al. ${ }^{40}$ 


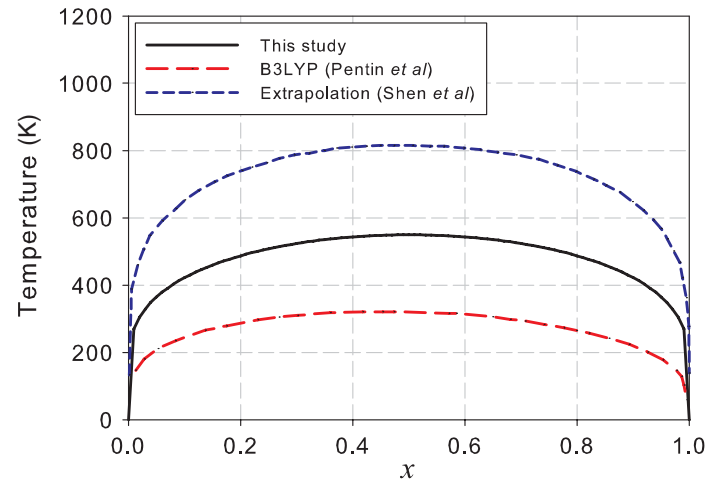

FIG. 4. (Color online) Phase diagram for the GaAs-InAs system showing the low-temperature miscibility gap. Our data are compared to the DFT-B3LYP simulations of Pentin et al..$^{42}$ and data extrapolated from the liquid-solid equilibria of Shen et al..$^{43}$

$0 \leqslant x \leqslant 1$ ) of $\mathrm{Ga}$ on a mole of $4 a$ lattice sites. Setting $\Delta G$ to zero and solving for $T$ from Eq. (5), it is possible to determine the critical temperature defining the limit of the $\operatorname{In}_{x} \mathrm{Ga}_{1-x}$ As phase. The low-temperature phase diagram showing the miscibility gap in $\operatorname{In}_{x} \mathrm{Ga}_{1-x}$ As is given in Fig. 4 where our data are compared with similar data from the literature. The results suggest that $\operatorname{In}_{x} \mathrm{Ga}_{1-x}$ As will be stable across the entire compositional range at temperatures greater than $550 \mathrm{~K}$. Figure 4 shows that our results fall between the previous DFT-B3LYP simulations of Pentin et al. $^{42}$ and the experimental data of Shen et al. ${ }^{43}$ that were extrapolated from thermodynamic functions obtained close to the liquidsolid interface. Pentin et ll $^{42}$ argue that such extrapolations are frequently unreliable while acknowledging that their DFT simulations ignore any vibrational contribution to the enthalpy.

The observation that $E\left(\operatorname{In}_{x} \mathrm{Ga}_{1-x} \mathrm{As}\right)>E(\mathrm{InAs})+$ $E(\mathrm{GaAs})$ has implications for the determination of the chemical potential of the atoms in the alloy since the chemical potentials of InAs or GaAs in $\mathrm{In}_{x} \mathrm{Ga}_{1-x} \mathrm{As}$ should not exceed the chemical potentials of the bulk binary alloys. Consequently, the chemical potentials of the atoms in $\operatorname{In}_{x} \mathrm{Ga}_{1-x}$ As are determined with reference to the elemental states only by assuming $\mu_{\mathrm{In}_{x} \mathrm{Ga}_{1-x} \mathrm{As}}=$ $x \mu_{\mathrm{In}}+(1-x) \mu_{\mathrm{Ga}}+\mu_{\mathrm{As}}$. The chemical potentials of the atoms may not exceed that of the bulk element; therefore, taking As as an example, $\mu_{\mathrm{As}}^{\text {rich }}=\mu_{\mathrm{As}(\mathrm{bulk})}$ represents the upper bound of the chemical potential. Continuing with As as our example, the lower bound (corresponding to As-poor growth conditions) can be determined by assuming the growth conditions were rich in both $\mathrm{In}$ and $\mathrm{Ga}$, i.e., $\mu_{\mathrm{As}}^{\text {poor }}=\mu_{\mathrm{In}_{x} \mathrm{Ga}_{1-x} \mathrm{As}}-\left[x \mu_{\mathrm{In}(\text { bulk })}+(1-x) \mu_{\mathrm{Ga}(\text { bulk })}\right]$.

\section{As vacancy defects in $\operatorname{In}_{x} \mathrm{Ga}_{1-x} \mathrm{As}$}

\section{Size effects}

For intermediate compositions of $\operatorname{In}_{x} \mathrm{Ga}_{1-x}$ As and InAs the arsenic vacancy formation energies are determined using the 64-atom supercells only. The local distortions of the arsenic sublattice due to the random arrangement of the In and $\mathrm{Ga}$ atoms on the $4 a$ Wyckoff sites means that each of the As sites in the simulation supercell is potentially unique. For
$\mathrm{In}_{0.25} \mathrm{Ga}_{0.75} \mathrm{As}$ and $\mathrm{In}_{0.75} \mathrm{Ga}_{0.25} \mathrm{As}$, all 32 sites are unique but for the $\mathrm{In}_{0.5} \mathrm{Ga}_{0.5}$ As only 16 As sites are unique as it is constructed from a 32-atom supercell. Consequently, it is necessary to perform a total of 80 spin-polarized defect simulations. Employing a larger SQS supercell would increase the computational overhead for each individual simulation but more importantly it would lead to a dramatic increase in the number of permutations that would have to be studied. However, the influence of supercell size on the formation energy and, in particular, on the configuration of the arsenic vacancy in GaAs can sometimes be significant. ${ }^{6}$ El-Mellouhi and Mousseau ${ }^{6}$ observe a Jahn-Teller distortion around the $\mathrm{V}_{\mathrm{As}}^{-1}$ defect in their 216 atom supercell, however, in their 64-atom supercell they found a $C_{2 v}$ symmetry. Clearly, it is important that the magnitude of any possible errors relating to the use of a 64-atom supercell are understood. Therefore, an arsenic vacancy defect was introduced into both 64 and 216 atom GaAs supercells and the supercells were minimised under constant pressure conditions (i.e., atoms positions and lattice vectors were allowed to change) and the formation energies and the symmetry of the surrounding $\mathrm{Ga}$ atoms were determined using MATERIALS STUDIO. ${ }^{44}$

In the nondefective GaAs structure the separation between two nearest-neighbor $\mathrm{Ga}$ atoms is predicted to be $4.06 \AA$, in good agreement with the experimental value of $4.00 \AA$. Two simulations were performed for the charge-neutral defect in the 64-atom supercell, one in which all of the atoms in the supercell are left on their perfect lattice positions and another where each atom is slightly displaced in a random direction. For the simulation where the atoms are left on their perfect lattice sites the separations between the four Ga atoms surrounding the arsenic vacancy are all reduced to $3.73 \AA$, maintaining a perfect tetrahedral site symmetry. Conversely, when the atoms initial positions are distorted the final symmetry of the surrounding Ga atoms can be best approximated to a $D_{2 d}$ point group. The maximum deviation of the atoms from a perfect $D_{2 d}$ structure was $0.04 \AA$; however, the deviation from a perfect $T_{d}$ symmetry was only $0.044 \AA$. This shows that while the symmetry has changed from $T_{d}$ to $D_{2 d}$ the distortion is not large.

For the simulation of the $\mathrm{V}_{\mathrm{As}}^{0}$ defect in a 216 atom supercell the atoms were randomly displaced from their perfect lattice sites. After minimization two of the separations between Ga atoms were reduced to $3.93 \AA$ and the remaining four were reduced to $4.04 \AA$, representing a shift to a JahnTeller $D_{2 d}$ symmetry with a maximum deviation of $0.019 \AA$. Clearly, the move to a larger supercell allows the $\mathrm{Ga}$ atoms surrounding the vacant $4 c$ site to move closer to a perfect $D_{2 d}$ structure.

The $\mathrm{V}_{\mathrm{As}}^{0}$ defect formation energies in the As-rich limit were $3.22,3.23$, and $3.33 \mathrm{eV}$ for the 64-atom undistorted, 64-atom distorted, and 216-atom supercells, respectively, compared to a value of $3.25 \mathrm{eV}$ from El-Mellouhi and Mousseau. ${ }^{6}$ These results show that for the charge-neutral defect the 64-atom supercell gives formation energies in good agreement with the larger supercell and is also able predict the same symmetry around the defect. We note that for intermediate $\mathrm{In}_{x} \mathrm{Ga}_{1-x}$ As compositions the perfect $T_{d}$ symmetry of each lattice site is broken due to the quasirandom distribution of the In and $\mathrm{Ga}$ atoms on the $4 a$ sublattice; therefore, the presence of a Jahn-Teller distortion surrounding the vacancy 

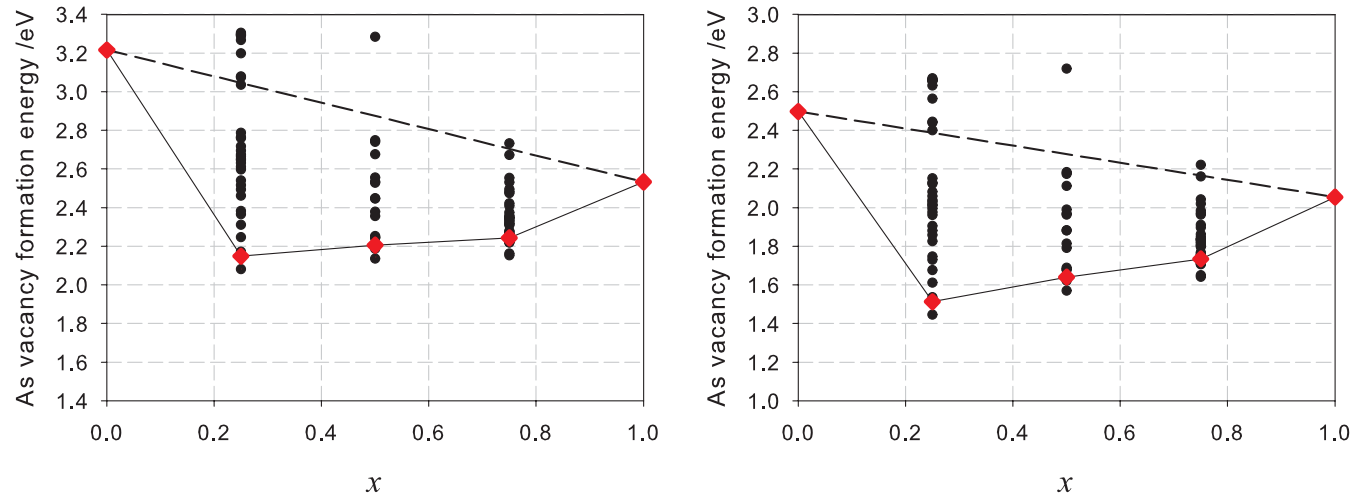

FIG. 5. (Color online) Plots of the defect formation energy of a $\mathrm{V}_{\mathrm{As}}^{0}$ defect in $\operatorname{In}_{x} \mathrm{Ga}_{1-x} \mathrm{As}$ under As-rich (a) and As-poor (b) conditions. Red diamonds represent configurationally averaged formation energies at $1000 \mathrm{~K}$.

would be swamped by the perturbation due the differing local environments.

\section{Defect formation energies}

Figures 5(a) and 5(b) show plots of the formation energies of charge-neutral As vacancy defects in As-rich and -poor growth conditions, respectively. The formation energy of the charge-neutral As vacancy, $\mathrm{V}_{\mathrm{As}}^{0}$, in GaAs has already been discussed in Sec. III C 1. The formation energy for this defect in InAs was found to be 2.53 and $2.06 \mathrm{eV}$ under As-rich and -poor growth conditions, respectively. Van Vechten ${ }^{5}$ predicted a value of $2.07 \mathrm{eV}$ in a stoichiometric sample and Hoglund et al. ${ }^{16}$ report a value of $>2.6 \mathrm{eV}$ under As-rich conditions.

As discussed previously, the adoption of SQS supercells allows a sampling of the different local environments on the disordered sublattice. This quasirandom distribution of ions allows for local relaxation effects to be modeled and gives rise to the distribution in the values of the defect formation energies shown in Figs. 5(a) and 5(b) for intermediate compositions of $\operatorname{In}_{x} \mathrm{Ga}_{1-x}$ As. Figures 5(a) and 5(b) clearly illustrate the significant variation $(\leqslant 1.2 \mathrm{eV})$ in defect formation energies due to different local environments in the random alloys. A typical formation energy for a neutral As vacancy defect was then determined by configurational averaging using Eq. $(7)^{45}$ as follows:

$$
\langle A\rangle=\frac{\sum_{c}^{C} A_{c} \exp ^{-\left(\frac{E_{\text {cell }}^{c}}{k_{B} T}\right)}}{\sum_{c}^{C} \exp ^{-\left(\frac{E_{\text {cll }}^{C}}{k_{B} T}\right)}},
$$

where $\langle A\rangle$ is the configurationally average, $A_{c}$ is the property being averaged for configuration $c, E_{\text {cell }}^{c}$ is the defect formation energy for configuration $c$, and $T$ is the temperature (for this study a temperature of $1000 \mathrm{~K}$ was adopted). The configurationally averaged defect formation energies are included as the (red) diamonds in Figs. 5(a) and 5(b).

As a first approximation the formation energy of $\mathrm{V}_{\mathrm{As}}^{0}$ in an intermediate $\operatorname{In}_{x} \mathrm{Ga}_{1-x}$ As compound can be estimated from a linear interpolation of the vacancy formation energies determined for the end member binary alloys; this is represented by the dashed line in Figs. 5(a) and 5(b). Clearly, the configurationally averaged formation energies are significantly lower than would be expected from such an interpolation and, indeed, are lower than for the end-member binary alloys.
By considering the detailed local relaxations it is possible to understand why this behavior occurs. At intermediate compositions one of the four surrounding atoms undergoes relaxation toward the now-vacant site, creating a quasi- $\mathrm{V}_{\mathrm{As}}-\mathrm{In} / \mathrm{Ga}_{i}-\mathrm{V}_{\mathrm{In} / \mathrm{Ga}}$ defect cluster. In the end-member binary alloys none of the surrounding atoms undergo this relaxation and the four atoms remain approximately equidistant from the vacancy. It is this difference in response to the introduction of the vacancy that allows the intermediate $\operatorname{In}_{x} \mathrm{Ga}_{1-x}$ As compounds to have lower defect formation energies than present in the end members. The atom that undergoes the greatest relaxation toward the vacancy was always found to be an In atom except when there were no In atoms immediately surrounding the vacancy. Configurations in which there are no In atoms surrounding the defect were found to have the highest formation energies, as illustrated in Fig. 6 where the defect formation energy is plotted as a function of the number of In nearest neighbors. Thus, as the number of In atoms surrounding the vacancy increases the formation energy of the $\mathrm{V}_{\mathrm{As}}^{0}$ defect decreases until there are three In atoms surrounding the vacancy. The defect formation energy when there are four In atoms surrounding the vacancy is then similar to the value obtained for three In atoms. As the concentration of InAs in $\operatorname{In}_{x} \mathrm{Ga}_{1-x}$ As increases the average number of indium atoms surrounding any $4 c$ lattice site will increase and, hence, the configurationally averaged formation

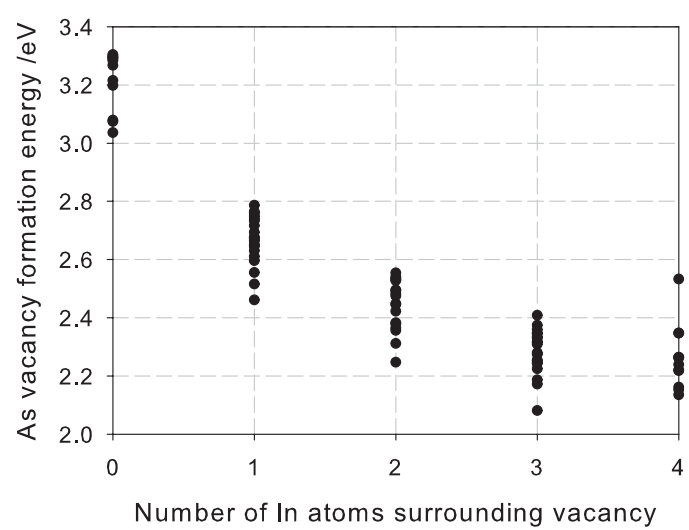

FIG. 6. Plot showing the formation energy of a $\mathrm{V}_{\mathrm{As}}^{0}$ defect in $\mathrm{In}_{x} \mathrm{Ga}_{1-x}$ As as a function of the number of In atoms located on nearest-neighbor $4 a$ lattice sites surrounding the vacancy. 


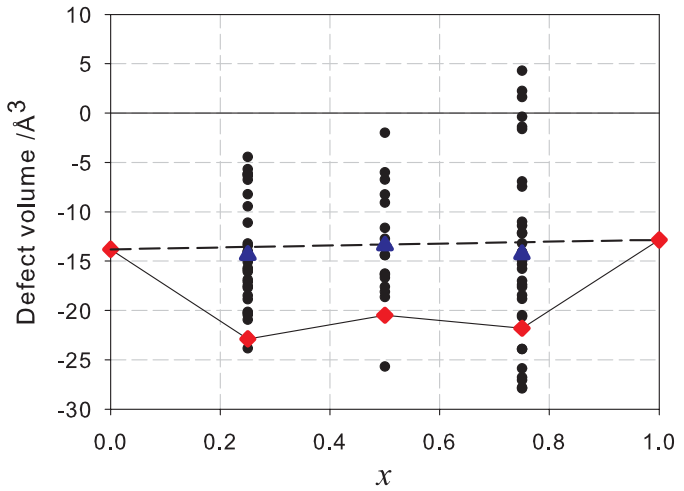

FIG. 7. (Color online) Defect volume resulting from the introduction of a $\mathrm{V}_{\mathrm{As}}^{0}$ defect in $\mathrm{In}_{x} \mathrm{Ga}_{1-x}$ As as a function of the concentration of InAs, i.e., $x$. Red diamonds represent the configurationally averaged values and blue triangles represent the mean values.

energy for $x=0.75$ and $x=0.5$ would be expected to be lower than for $x=0.25$. Figures 5(a) and 5(b) suggest that this is not the case. The reason is that the configurations with three In atoms surrounding the vacancy for $x=0.25$ have a particularly low energy due to the increased relaxation of one of the indium atoms toward the vacant site, lower than that observed for $x=0.5$ and $x=0.75$. One explanation for this is that the high concentration of smaller Ga atoms in the second nearest neighbor $4 a$ lattice sites allows the larger In atom to move further into the space created by the removal of the As atom, thus reducing the strain in the lattice.

\section{Defect volumes}

Finally, we investigate the volume change resulting from the introduction of a $\mathrm{V}_{\mathrm{As}}^{0}$ defect into $\mathrm{In}_{x} \mathrm{Ga}_{1-x}$ As. The defect volume here is defined as the volume of the defective supercell minus that of the perfect supercell. Figure 7 shows the defect volume versus the concentration of $\operatorname{InAs}$ in $\operatorname{In}_{x} \mathrm{Ga}_{1-x}$ As. The mean and configurationally averaged defect volumes have also been determined and are shown in Fig. 7.

The values obtained for the defect volume display a large variation similar to that observed for the formation energies; however the mean defect volumes for the chargeneutral As vacancy provide a good match to the linear interpolation of the defect volumes in the end members. Conversely, the magnitude of the configurationally averaged defect volumes are significantly greater than both the average and the values predicted from a linear interpolation. This shows that, in general, the larger the change in the cell size on incorporation of a $\mathrm{V}_{\mathrm{As}}^{0}$ defect, the lower the defect formation energy. Therefore, the configurationally averaged defect volume is weighted toward the larger volume changes due their being lower in energy and so more thermodynamically favorable.

\section{CONCLUSIONS}

Electronic structure simulations have been used in conjunction with SQS supercells to investigate the structure and phase stability of $\operatorname{In}_{x} \mathrm{Ga}_{1-x}$ As. Our results suggest the lattice parameters of the intermediate $\operatorname{In}_{x} \mathrm{Ga}_{1-x}$ As compositions are lower than would be expected by taking a linear interpolation of the lattice parameters of the end members, i.e., there is a small negative deviation from Vegard's law in agreement with previous simulations. ${ }^{25}$ The atomization energies of the solid $\mathrm{In}, \mathrm{Ga}, \mathrm{As}$, and $\mathrm{GaAs}$ are in excellent agreement with the literature values, indicating that the GGA-PBE exchange correlation functional is adequate for determining energetic properties. We have determined the $0 \mathrm{~K}$ atomization energies across the compositional range of $\operatorname{In}_{x} \mathrm{Ga}_{1-x}$ As and observed a negative deviation from a linear interpolation of the atomization energies of GaAs and InAs. Consequently, we predict a positive enthalpy of mixing for $\operatorname{In}_{x} \mathrm{Ga}_{1-x}$ As from InAs and GaAs, and, therefore, the presence of a miscibility gap, that would correspond to the low-temperature region of the GaAs-InAs phase diagram. $\operatorname{In}_{x} \mathrm{Ga}_{1-x}$ As is, however, expected to be thermodynamically stable at temperatures greater than $550 \mathrm{~K}$. The relatively low temperature range at which $\operatorname{In}_{x} \mathrm{Ga}_{1-x}$ As is expected to undergo spinoidal decomposition may be the reason this has not been observed experimentally. Miscibility gaps are, however, common in other III-V materials, ${ }^{46}$ such as $\mathrm{Ga}_{x} \mathrm{In}_{1-x} \mathrm{P}^{47} \mathrm{GaP}_{x} \mathrm{Sb}_{1-x},{ }^{47}$ $\mathrm{InP}_{x} \mathrm{Sb}_{1-x},{ }^{47}$ and $\operatorname{InAs}_{x} \mathrm{Sb}_{1-x} .{ }^{47,48}$

The formation energies for the introduction of a $\mathrm{V}_{\mathrm{As}}^{0}$ defect under As-rich conditions in GaAs and InAs were predicted to be 3.31 and $2.53 \mathrm{eV}$, respectively. These values are in excellent agreement with previously determined values of $3.25 \mathrm{eV}$ for $\mathrm{GaAs}^{6}$ and $>2.6 \mathrm{eV}$ for InAs. ${ }^{16}$ For intermediate values of $x$ there is a large variation in the formation energies and defect volumes of the charge-neutral arsenic vacancy defect: This arises due to the random arrangement of $\mathrm{In}$ and $\mathrm{Ga}$ atoms on the $4 a$ sublattice. As a consequence of the random arrangement of In and $\mathrm{Ga}$ atoms an As vacancy may be surrounded by between zero and four In atoms. As the number of In atoms surrounding an arsenic vacancy increases, the formation energy of the vacancy decreases (see Fig. 6). The formation energy of a $\mathrm{V}_{\mathrm{As}}^{0}$ defect in $\operatorname{In}_{0.25} \mathrm{Ga}_{0.75}$ As surrounded by three atoms was found to be particularly low due to the propensity for one of the In atoms to move closer to the vacant arsenic site than was observed in $\mathrm{In}_{0.5} \mathrm{Ga}_{0.5}$ As and $\mathrm{In}_{0.75} \mathrm{Ga}_{0.25}$ As. It is these low-energy configurations that cause the apparent distortion in the configurationally averaged formation energies shown in Figs. 5(a) and 5(b) at $x=0.25$. Across the entire compositional range the configurationally averaged formation energies for the $\mathrm{V}_{\mathrm{As}}^{0}$ defect were found to be significantly lower than would be estimated from a linear interpolation of the end-member binary alloys, thereby demonstrating the necessity of performing a rigorous study of the different defect configurations in random alloys such as $\mathrm{In}_{x} \mathrm{Ga}_{1-x}$ As.

\section{ACKNOWLEDGMENTS}

This paper was based on work supported in part by King Abdullah University of Science and Technology (KAUST). Computational resources were provided by the Imperial College High Performance Computing Service (http://www.imperial.ac.uk/ict/services/teachingandresearchservices/highperformancecomputing). 
*samuel.murphy05@ic.ac.uk

${ }^{1}$ A. M. Sonnet, C. L. Hinkle, M. N. Jivani, R. A. Chapman, G. P. Pollack, R. M. Wallace, and E. M. Vogel, Appl. Phys. Lett. 93, 122109 (2008).

${ }^{2}$ C. L. Hinkle, M. Milojevic, E. M. Vogel, and R. M. Wallace, App. Phys. Lett. 95, 151905 (2009).

${ }^{3}$ N. Dharmarasu et al., Appl. Phys. Lett. 79, 2399 (2001).

${ }^{4}$ V. P. Kunets, T. A. Morgan, Y. I. Mazur, V. G. Dorogan, P. M. Lytvyn, M. E. Warw, D. Guzun, J. L. Shultz, and G. J. Salamo, J. Appl. Phys. 104, 103709 (2008).

${ }^{5}$ J. A. Van Vechten, J. Electrochem. Soc. 122, 419 (1975).

${ }^{6}$ F. El-Mellouhi and N. Mousseau, Phys. Rev. B 71, 125207 (2005).

${ }^{7}$ P. A. Schultz and O. Anatole von Lilienfeld, Modell. Simul. Mater. Sci. Eng. 17, 84007 (2009).

${ }^{8}$ K. Laasonen, R. M. Nieminen, and M. J. Puska, Phys. Rev. B 45, 4122 (1992).

${ }^{9}$ F. Corsetti and A. A. Mostofi, Phys. Rev. B 84, 035209 (2011).

${ }^{10}$ M. J. Puska, S. Poykko, M. Pesola, and R. M. Nieminen, Phys. Rev B 58, 1318 (1998).

${ }^{11}$ B.-H. Cheong and K. J. Chang, Phys. Rev. B 49, 17436 (1994).

${ }^{12}$ S. Poykko, M. J. Puska, and R. M. Nieminen, Phys. Rev. B 53, 3813 (1996).

${ }^{13}$ H. Seong and L. J. Lewis, Phys. Rev. B 52, 5675 (1995).

${ }^{14}$ R. W. Jansen and O. F. Sankey, Phys. Rev. B 39, 3192 (1989).

${ }^{15}$ H.-P. Komsa and A. Pasquarello, Microelectron. Eng. 88, 1436 (2011).

${ }^{16}$ A. Hoglund, C. W. M. Castleton, M. Gothelid, B. Johansson, and S. Mirbt, Phys. Rev. B 74, 075332 (2006).

${ }^{17}$ W. P. Gillin, S. S. Rao, I. V. Bradley, K. P. Homewood, A. D. Smith, and A. T. R. Briggs, Appl. Phys. Lett. 63, 797 (1993).

${ }^{18}$ M. C. Payne, M. P. Teter, D. C. Allan, T. A. Arias, and J. D. Joannopoulos, Rev. Mod. Phys. 64, 1045 (1992).

${ }^{19}$ S. Clark, M. Segall, C. Pickard, P. Hasnip, K. Refson, and M. Payne, Z. Kristallogr. 220, 1045 (2005).

${ }^{20}$ J. P. Perdew and A. Zunger, Phys. Rev. B 23, 5048 (1981).

${ }^{21}$ H. J. Monkhorst and J. D. Pack, Phys. Rev. B 13, 5188 (1976).

${ }^{22}$ S. T. Murphy, P. Zeller, A. Chartier, and L. Van Brutzel, J. Phys. Chem. C 115, 21874 (2011).

${ }^{23}$ A. Zunger, S. H. Wei, L. G. Ferreira, and J. E. Bernard, Phys. Rev. Lett. 65, 353 (1990).

${ }^{24}$ K. C. Hass, L. C. Davis, and A. Zunger, Phys. Rev. B 42, 3757 (1990).
${ }^{25}$ S. T. Murphy, A. Chroneos, C. Jiang, U. Schwingenschlögl, and R. W. Grimes, Phys. Rev. B 82, 073201 (2010).

${ }^{26}$ A. Chroneos, H. Bracht, C. Jiang, B. P. Uberuaga, and R. W. Grimes, Phys. Rev. B 78, 195201 (2008).

${ }^{27}$ S. B. Zhang and J. E. Northrup, Phys. Rev. Lett. 67, 2339 (1991).

${ }^{28}$ S. Lany and A. Zunger, Phys. Rev. B 78, 235104 (2008).

${ }^{29}$ H. E. Swanson and R. K. Fuyat, Z. Anorg. Allg. Chem. 286, 118 (1956).

${ }^{30}$ R. C. Weast, CRC Handbook of Chemistry and Physics (CRC Press, Boca Raton, FL, 1984).

${ }^{31}$ C. E. Weir, G. J. Piermarini, and S. Block, J. Phys. Chem. 54, 2768 (1971).

${ }^{32}$ M. W. Chase Jr., C. A. Davies, J. R. Downey, D. J. Frurip, R. A. McDonald, and A. N. Syverud, NIST JANAF Thermochemical Tables 1985 (National Institute of Standards and Technology, Washington, DC, 1986).

${ }^{33}$ D. Schiferl and C. S. Barrett, J. Appl. Crystallogr. 2, 30 (1969).

${ }^{34}$ L. Vegard, Z. Phys. 5, 17 (1921).

${ }^{35}$ I. Katayama, T. Nakai, T. Inomoto, and Z. Kozuka, T. Jpn. I. Met. 30, 354 (1989).

${ }^{36}$ N. A. Goryunova and N. N. Fedorova, Zh. Tekh. Fiz. 25, 1339 (1955).

${ }^{37}$ T. S. Liu and E. A. Peretti, T. Am. Soc. Met. 45, 677 (1953).

${ }^{38}$ K. J. Schulz, O. A. Musbah, and Y. A. Chang, J. Phase Equilib. 12, 10 (1991).

${ }^{39}$ J. Paier, M. Marsman, and G. Kresse, J. Chem. Phys. 127, 024103 (2007).

${ }^{40}$ B. C. Rugg, N. J. Silk, A. W. Bryant, and B. B. Argent, Calphad 19, 389 (1995).

${ }^{41}$ D. A. Porter and K. E. Easterling, Phase Transitions in Metals and Alloys (CRC Press, Boca Raton, FL, 2004).

${ }^{42}$ I. V. Pentin, J. C. Schon, and M. Jansen, Phys. Chem. Chem. Phys. 12, 8491 (2010).

${ }^{43}$ J. Y. Shen, C. Chatillon, I. Ansara, A. Watson, B. Rugg, and T. Chart, CALPHAD 19, 215 (1995).

${ }^{44}$ Accelrys, MATERIALS STUDIO [http://accelrys.com].

${ }^{45}$ I. T. Todorov, N. L. Allan, M. Y. Lavrentiev, C. L. Freeman, C. Mohn, and J. A. Purton, J. Phys. Condens. Matter 16, S2751 (2004).

${ }^{46}$ G. B. Stringfellow, J. Cryst. Growth 65, 454 (2002).

${ }^{47}$ K. Ishida, T. Nomura, H. Tokunaga, H. Ohtani, and Nishizawa, J. Less-Common Met. 155, 193 (1989).

${ }^{48}$ H. Miyoshi and H. Horikoshi, J. Cryst. Growth 227, 571 (2001). 